Departamento de Psiquiatría, Escuela de Medicina, Universidad de Valparaíso. Valparaíso, Chile.

Conflictos de intereses: nada que declarar.

Recibido el 4 de noviembre de 2012, aceptado el 11 de noviembre de 2013 Correspondencia a: Dr. Gustavo Figueroa. gfigueroacave@gmail.com

\section{La seducción sexual infantil: revolución y repercusiones de la teoría de Freud}

\author{
GUSTAVO FIGUEROA C.
}

\section{The infantile sexual seduction: revolution and aftermath of Freud's theory}

There is no question about the negative effects of child sexual abuse. Freud's seduction theory asserts that psychoneuroses in adults are caused by reactivation of forgotten recollections of gross sexual abuse (involving the genitals) that had taken place prior to the age of 8 to 10 years. His contribution consisted in the discovery of specific events, prior to puberty, which were indispensable to the formation of psychoneuroses. If an adult patient recalled an infantile sexual experience, Freud assumed the interference of a pervert: a child was sexually innocent unless it had been traumatized. But Freud's technique of clinical exploration had not attained adequate reliability and was not immune to prejudices. Freud himself dropped his mechanical, static theory that presupposed a single type of accidentally occurring trauma prior to puberty, allowing him to develop his new drive and fantasy theory.

(Rev Med Chile 2014; 142: 84-89)

Key words: Child abuse, sexual; Incest; Freudian theory.

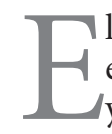
l abuso sexual infantil exhibe una prevalencia en la población general que oscila entre 3\% y $33 \%$ y se encuentra extendido a todos los países, culturas y estratos sociales ${ }^{1}$. Quizás estas cifras subestiman su existencia real por su baja denuncia $^{2}$, pero constituyen un porcentaje considerable (13\%-26\%) en la práctica de la medicina de atención primaria ${ }^{3}$. Metanálisis recientes dan cuenta de una relación entre abuso y trastornos depresivos, de angustia, del comer, del dormir, estrés postraumático, abuso de sustancias y alcohol e intento de suicidio, independientes del sexo y edad de ocurrido el abuso ${ }^{4-6}$. Por otra parte, los problemas metodológicos son importantes y no se han podido resolver satisfactoriamente hasta ahora: falta de claros criterios internacionales de operacionalización ${ }^{7}$, diversas escalas de evaluación, diferencias entre autoinformes y entrevistas por terceros, variación de acuerdo al número de preguntas, diferencia entre conductas y estados subjetivos, no distinción entre aspectos psicológico y legal, intereses de los investigadores y revistas especializadas ${ }^{8,9}$. Sin em- bargo, el principal cuestionamiento ha surgido al ponerse en duda la veracidad de los informantes o la realidad de los recuerdos ${ }^{10}$, duda que pone en tela de juicio los estudios que se han acumulado con velocidad creciente en los últimos años ${ }^{11}$ y que generó el concepto "síndrome de falso recuerdo" 12 .

Hacia 1896 Freud elaboró una hipótesis revolucionaria que se conoció como "teoría de la seducción sexual infantil". Esta investigación desencadenó encendidas polémicas por las consecuencias para la vida sexual de los niños, las implicancias para el funcionamiento afectivo al interior de familias y grupos de socialización y suspicacias acerca de las intenciones del comportamiento de adultos y pequeños ${ }^{13-15}$.

El presente trabajo investiga tres aspectos de la teoría de Freud para comprender la relación entre hecho, recuerdo y fantasía: la estructura interna de la teoría, los motivos de su posterior abandono por parte de Freud y las repercusiones que ha ejercido sobre la concepción actual de los abusos sexuales tempranos. 


\section{La teoría de la seducción sexual infantil}

Sigmund Freud (1856-1939) constituye un referente determinante en la concepción de la sexualidad en el mundo contemporáneo. Él provocó un giro decisivo: la sexualidad adquirió un rango antropológico básico al constituirse en fundamento último de las motivaciones humanas, sus variaciones permitieron entender diversos cuadros mentales, la conducta normal se comprendió a partir de impulsos eróticos ocultos y su existencia se manifiesta desde el primer día del nacimiento; esto es, nuestra condición es ser sexuado y no sólo portador de un sexo ${ }^{16}$.

Para su propia sorpresa, apenas ingresado en la clínica, Freud descubrió a la sexualidad como causa primaria de cuadros psicológicos y con ello impugnó a la psiquiatría tradicional. En apenas ocho años elaboró una nosología original basada en la etiología, superando a la oficial sustentada en la sintomatología. Diferenció dos grupos de afecciones: las "neuropsicosis de defensa" -la histeria, fobias, obsesiones, algunos cuadros paranoides, producto de afectos sexuales reprimidos por fuerzas psíquicas que se oponen activamente a su conocimiento consciente ${ }^{17}$, y "las neurosis reales (presentes)" (aktuelle Neurosen) -la neurastenia y neurosis de angustia-, generadas a partir de prácticas sexuales erradas o aberrantes (masturbación y coitus interruptus $)^{18}$.

Pero las sorpresas no se detuvieron. A comienzos de 1896 -se extendió hasta 1897- se produjo otro cambio brusco, consecuencia de la necesidad tanto de precisar más finamente la etiología sexual como de comunicaciones espontáneas de sus pacientes. En tres artículos Freud propuso su nueva teoría que trajo aparejada una conceptualización que resultó casi más revolucionaria, pero que esta vez terminó remeciéndolo de manera personal ${ }^{19-21}$. Aceptó la herencia como causa predisponente, según era clásico en psiquiatría, y las causas concurrentes: emociones morales, agotamiento intelectual, enfermedades agudas, accidentes, intoxicaciones. Pero las causas específicas que había postulado -deseos eróticos, relaciones ilícitas, presiones para intimar sexualmente-, sucesos que habían sido reprimidos y no descargados, por más vergonzosos y prohibidos que fueran a primera vista, se le mostraron como absolutamente inespecíficos. Si los "histéricos sufren de reminiscencias" 22 , estos recuerdos eran solamente afectos fuertes y bruscos, con un contenido comprensible cuya relación era demasiado evidente, superficial y obvia tanto con la biografía como con el sentido de los síntomas.

Los nuevos hechos clínicos, producto "de cien o más horas de trabajo", le entregaron: a) la especificidad etiológica que faltaba y b) lo oculto que estaba embozado detrás de las manifestaciones inmediatas. Las pacientes comenzaron a relatarle espontáneamente sucesos acaecidos en su infancia antes de los diez años y aún previo a los cuatro "cuando todavía eran mecidas en los brazos", lo cual condujo a Freud a postular la intrusión de un pervertido: una niña era sexualmente inocente a menos que hubiese sido traumatizada. Eran imposiciones externas, que se podían dividir en tres tipos. Primera, "experiencias sexuales violentas que afectan el propio cuerpo -aún relación sexual (en un sentido amplio)", semejante a un coito, con "excitación o estimulación real de los genitales, que desembocan en un abuso sexual (Mißbrauch)" o un intento de violación (Vergewaltigung) y que de "golpe descubre la brutalidad del placer genital". Segunda, ser testigo involuntario "del acto sexual de los padres, acto que irrumpe como algo horrible y que hiere el sentido moral infantil"; $y$, tercera, vivencias curiosamente insignificantes, como ser rozada en los muslos por casualidad, escuchar ruidos, palabras de doble sentido o insinuaciones equívocas, pero que permiten intuir de manera indirecta la posibilidad de algo prohibido, obsceno. "En la mayoría de los casos encontré que dos o tres de estas etiologías estaban actuando conjuntamente". Los abusadores eran adultos de muy diferente condición: "institutrices, empleadas domésticas, tutores, o, desgraciadamente muy frecuentemente, parientes cercanos", como hermanos, además de "empleados de servicio, profesores, o personas encargadas del cuidado de la menor". En síntesis, las niñas, como criaturas sexualmente puras, habían sido víctimas de tres tipos de traumas: atentados, esto es, abusos aislados, únicos, "por adultos que eran extraños", que originaban una intensa sensación de espanto; relaciones regulares, de larga duración con un sujeto mayor "que iniciaba a la pequeña en la actividad genital"; $y$ "relaciones sexuales entre dos niños de diferentes sexo, especialmente hermano y hermana". Estos descubrimientos implicaban el siguiente corolario: la ocurrencia de traumas sexuales después de la pubertad no es causante de histeria o psiconeurosis 
en sentido estricto, sino sólo de cuadros emocionales o reactivos porque el aparato psíquico ya ha alcanzado un grado de madurez suficiente como para comprender y manejar los impulsos eróticos de una manera distinta.

Sin embargo, estas experiencias infantiles necesitaban de una condición indispensable: debían ser reprimidas, "impulsadas fuera de la conciencia", para que se convirtieran en patógenas en la vida adulta porque, si permanecían conscientes, no se originaba la neurosis. Lo decisivo es que estos recuerdos inconscientes, "ocurridos durante el período de inmadurez sexual", a pesar de haber sido olvidados en apariencia, "tienen una conexión lógica o asociativa” con los síntomas neuróticos posteriores. Con lo que resulta que la cadena causal es bastante más compleja que una simple relación lineal entre trauma precoz y aparición de síntomas. La memoria del abuso sexual temprano permanece olvidada durante la infancia hasta que la excitación generada por el despertar de la pubertad carga afectivamente a los recuerdos ligados a los antiguos sucesos traumáticos. Vale decir, para que estos se tornen patógenos se necesita que se estimulen por un segundo trauma en los años post puberales. Este tipo de "relación invertida" entre el efecto psíquico del recuerdo y el evento actual es el que explica el efecto diferido (Nachträglichkeit) del trauma infantil. En otros términos, no es el trauma per se el factor determinante sino la combinación de: 1) Un recuerdo traumático inconsciente; 2) Su gran intensificación por la maduración sexual puberal y 3) Un suceso post puberal de naturaleza provocativa que enciende la memoria y la pone en estado activo. Estos tres elementos son las precondiciones ineludibles para el desarrollo de una histeria en un adulto.

Freud se adelantó y rechazó tres objeciones posibles. La primera y segunda eran antagónicas: que el abuso sexual era poco frecuente en relación al gran número de pacientes histéricas y que el abuso sexual de las niñas estaba mucho más expandido que el trastorno histérico. Frente a la primera, recordó que existían prácticas sexuales ejecutadas por las cuidadoras "aún cuando las bebitas estaban en sus brazos"; frente a la segunda, "la excesiva frecuencia de un factor etiológico no es posible de ser usada como una objeción contra su significado etiológico". La tercera tocaba su método de investigación: haber sido poco cuidadoso al creer que lo que constituían recuerdos de sucesos reales, sólo correspondían a fantasías bona fide de sus enfermas. Freud negó rotundamente esta posibilidad porque no sólo ciertos abusos habían sido confirmados por terceros, sino porque él no tenía ideas preconcebidas o expectativas sobre los resultados obtenidos; además su técnica impedía cualquiera postura autoritaria: "antes que vinieran a análisis las pacientes, no sabían nada de estas escenas. Como regla, ellas se indignaban cuando se las advertía que estas escenas iban a emerger. Sólo la más fuerte compulsión (Zwang) del tratamiento las inducía a embarcarse en la reproducción de ellas".

Desde el 6 de diciembre de 1896, por un breve período y solamente en cartas privadas dirigidas a su amigo Flie $\beta$, aparece un nuevo y asombroso factor: el padre como perverso y uno de los principales involucrados en la etiología, afirmación que culmina el 8 de febrero de 1897: "Desgraciadamente, mi propio padre ha sido uno de los perversos y es el culpable (verschulden) de la histeria de mi hermano (cuyos malestares son, todos ellos, identificación) y de una hermana menor. La frecuencia de esta circunstancia me hace dudar a menudo"23. La última frase mitiga lo afirmado en la primera y demuestra su profunda ambivalencia ante el espinoso tema. Esto es, las evidencias recogidas hasta esos momentos son poco concluyentes para él mismo por ser indirectas, esbozadas y débiles: identificaciones entre hijo y padre al compartir perversiones aunque diferentes a la de la seducción, sensaciones nauseosas simbólicas similares entre hija y padre, rechazo excesivamente enfático por parte de las jóvenes a sus interpretaciones sobre seducciones, amenaza de término de la terapia por parte de Freud para que la paciente admita su interpretación. En su correspondencia personal, los argumentos destinados a probar el incesto paterno poseían un valor muy cambiante, a veces eran admitidos fácil e inequívocamente por él, para luego ser desechados de manera rotunda y emocional. Empero, esto no le impide exclamar ¡Habemus papam!, como si con ello quisiera dar a entender, de manera sarcástica, " $¡ L o$ tenemos al padre!".

\section{"Ya no creo más"}

El 21 de septiembre de 1897 modifica radicalmente su postura después de un sueño erótico que 
tuvo con su hija y el comienzo de su trascendental autoanálisis tras la muerte de su padre: "Ya no creo más en mi neurótica", lo cual implicaba la teoría del incesto paterno en un sentido estrecho y, en el amplio, toda la teoría de la psicogénesis traumática de las psiconeurosis ${ }^{24,25}$.

Freud entrega cuatro argumentos para su rechazo. Primero, fracaso en llevar a "término real" un solo tratamiento de sus 18 casos, "me parece como si faltara una pieza esencial en alguna parte", y mientras los pacientes no alcancen el final, "no me siento seguro y no puedo estar alegre". Segundo, no obtener un "éxito completo" que pudiera valer como mejoría, puesto que ni siquiera había conseguido una recuperación más allá de la desaparición sintomática parcial, esto es, ausencia de confirmación clínica. Tercero, discontinuaciones o abandonos abruptos de la psicoterapia, aún en los casos en que se estaban logrando progresos en el descubrimiento de los traumas. Cuarto, la mejoría fragmentaria del cuadro podía ser comprendida de manera "corriente", vale decir, no como "consecuencia específica de las interpretaciones y, por tanto, no constituyen una prueba inequívoca de la corrección de la teoría" y de la especificidad de la técnica ${ }^{26,27}$.

A esto se suman dos reflexiones. Por una parte, debió reconocer que la tajante aseveración "la sorpresa de que en cada uno de los casos el padre había de ser imputado de perverso, el mío propio incluido", no estaba basada en pruebas sino en inferencias, sin fundamento objetivo y constatable. Por otra, "la evidencia cierta de que en lo inconsciente no existe un signo de realidad, de manera que no se puede distinguir la verdad de la ficción cargada con afecto". Esta última adquirirá un valor tan especial que será decisiva para la formulación de su nueva teoría sobre la génesis de las psiconeurosis: opondrá la realidad externa a la realidad interna (fantasía), siendo esta última tan potente como la anterior.

La retractación fue clara pero su desenlace no resultó tan claro. Su desdecirse y rectificarse continuadamente delatan el importante compromiso emocional y personal con que se aferró a su teoría. Ya mencionamos que nunca comunicó científicamente la parte concerniente al incesto paterno. Además, en la correspondencia con Flie $\beta$ la impugnación definitiva recién ocurrió el 3 de enero de 1899, precedida de reiteradas defensas -aunque circunscritas- de su teoría. Públicamen- te, en 1898 guarda un obstinado silencio al tratar las psiconeurosis ${ }^{28}$, lo mismo que en sus importantes textos sobre los sueños $(1900)^{29}$ y la vida cotidiana $(1901)^{30}$. Su rechazo oficial lo esbozó escuetamente en 1905, aunque aclaró "no exageré la frecuencia o importancia (de la seducción en la niñez temprana)"31. Sólo al año siguiente vino la refutación inequívoca, empero previniendo que su material indudablemente "incluía desproporcionadamente un gran número de casos en que se había efectuado una seducción por un adulto o un niño mayor", esto es, aceptando la existencia real de violencia sexual durante la infancia ${ }^{32}$. Los escritos autobiográficos distorsionaron o esquivaron algunos hechos, pero hicieron presente la realidad indudable de los abusos tempranos ${ }^{33,34}$, al igual que en sus conferencias y patografías, donde confesó que hubo "invenciones" (erdichten), aunque "la seducción durante la infancia mantiene su importancia" 35,36 .

Lo notable es que en su último escrito, dedicado a Moisés, reaparece el trauma precoz en todo su vigor, ahora como configurador de la identidad del sujeto, trauma entendido no sólo onto sino filogenéticamente: ascendemos a lo humano a consecuencia del trauma originario de la humanidad ${ }^{37}$. Antes de morir dejó inacabada una vertiente novedosísima e insospechada: ya no es el padre el seductor original sino la madre, la que, por primera vez, estimula inevitablemente los genitales despertándole las sensaciones más placenteras y profundas, y "por el cuidado higiénico del cuerpo de la niña, se llega a constituir en la primera seductora" ${ }^{\prime 3}$.

\section{Acción-diferida (Nachträglichkeit)}

1. La teoría de la seducción sexual infantil de Freud buscó enfrentarse a los problemas de hecho, recuerdo y fantasía que acosan a la investigación actual sobre abuso infantil de un modo radicalmente diferente. Aunque resulta paradójico porque cumple más de un siglo, es novedoso e inédito por cuanto parte de otra perspectiva: la comprensión de los mecanismos psicológicos profundos, específicos y personales de su patogénesis. Para ello se adentra e interpreta a partir de los afectos que subyacen y explican a los recuerdos (reprimidos), hechos (disfrazados y distorsionados) y fantasía (eróticas). 
2. Plantea que traumas sexuales tempranos provocan trastornos emocionales en adultos por acción-diferida. Se opone a la concepción actual de trauma y que está presente en todas las investigaciones recientes porque se basan en el DSM-IV ${ }^{39}$, esto es, de una causalidad trauma -respuesta- pasado actuando como estresor determinante del presente. Freud propone explícitamente lo inverso: es el presente el que decide retroactivamente el pasado infantil. Las experiencias de abuso no tuvieron significación sexual para la niña sino posteriormente, recién cuando la pubertad le permitió comprender las caricias y acosos como propiamente eróticos. Estos recuerdos permanecen reprimidos hasta que una segunda vivencia posterior los estimula y les confiere su valor patógeno. Lo traumatizante no es simple excitación sino elaboración, conjunto de operaciones mentales profundas que configuran retrospectivamente la memoria hiper estimulada.

3. El recuerdo de la experiencia es el agente últimamente traumático. Pero Freud nunca pudo resucitar esas experiencias infantiles originales, a pesar de sus aseveraciones, sino sólo inferirlas; fue su trabajo de reconstrucción el que posibilitó descifrar y deducir su significado sexual. No existió re-vivir sino re-construir ${ }^{40}$.

4. Esta ausencia de recuerdo y, por tanto, de curación, motivó a Freud a rechazar su teoría y a entender la sexualidad como agente patógeno desde otra perspectiva. Surgió una nueva teoría sexual y con ello la revolucionaria concepción de la psiquis inconsciente: la "fantasía" psíquica y la "pulsión (instinto)" psicosexual.

5. En esta nueva concepción las "experiencias" traumáticas se entendieron como "fantasías" traumáticas, la "realidad externa" en proyección de la "realidad interna", la "verdad externa" en distorsión de la "verdad interna". Sin negar la existencia de experiencias de seducción efectivamente vividas, Freud captó ahora que las violaciones sexuales narradas no eran "mentiras" conscientes sino "verdades" inconscientes-no "engaños" sino "fantasías" producto de deseos, ilusiones, imaginaciones (Einbildungen) con intensísima fuerza patógena por provenir, en algunos casos, de esas vivencias realmente experimentadas.

6. La sexualidad también se configuró de manera radicalmente distinta: como "pulsión (instinto)" sexual (Sexualtrieb), impulso básico que se expresa desde el nacimiento mismo y sufre una compleja maduración que sólo en la pubertad termina organizándose bajo la primacía de la genitalidad. Por tanto, se trata de una constitución psicosexual propia del bebé, lo que excluye toda posibilidad de una infancia "inocente" o anterior a cualquiera seducción erótica o apetito perverso: es la parte oscura de nosotros-mismos. Sin embargo, la sexualidad, por ser inherente al ser humano, compromete a los adultos: padre, madre, parientes, extraños y ellos expresan estas tendencias sexuales en los niños seduciéndolos, acosándolos, violándolos.

7. El abuso sexual infantil es una realidad constatable en la sociedad, pero más complejo de lo que suponen las modernas investigaciones centradas en la medición objetiva, cuantitativa de la fiabilidad y validez, propias de la psiquiatría biológica. Freud no estuvo cerrado a esta posibilidad porque preveía que arribaría en un futuro no lejano; por su formación médica y clínica, pronosticó que sus trabajos eran preliminares ya que luego se traducirían y fundamentarían en sus componentes biológicos básicos. Pero fue modesto y se contentó con atenerse a la realidad que él comprobaba en sus pacientes. Así se limitó a hablar de serie complementaria cuando los factores determinantes de una condición patológica eran dos o más: para que surja el trastorno se requiere de ellos en distinta proporción, si uno es fuerte los otros pueden ser menos importantes $y$ viceversa. La seducción infantil se estructura de esa manera: desde el exterior -deseos incestuosos de padres y adultos- e interior-fantasías desiderativas, defensivas y proyectivas de las pulsiones ansiadas profundamente.

\section{Referencias}

1. Stoltenborgh M, van Ijzendoorn MH, Euser EM, Bakermans-Kranenburg MJ. A global perspective on child sexual abuse: meta-analysis of prevalence around the world. Child Maltreat 2011; 16: 79-110.

2. Back SE, Jackson JL, Fitzgerald M, Shaffer A, Salstrom S, Osman MM. Child sexual and physical abuse among college students in Singapore and the United States. Child Ab Neglect 2003; 27: 1259-75.

3. Cold J, Petruckevitch A, Chung WS, Richardson J, Moorey S, Feder, G. Abusive experiences and psychiatric morbidity in women primary care attenders. Brit J Psychiatry 2003; 183: 332-9.

4. Chen LP, Murad MH, Paras ML, Colbenson KM, Sattler 
$\mathrm{AL}$, Goranson EN, et al. Sexual abuse and lifetime diagnosis of psychiatric disorders: systematic review and meta-analysis. Mayo Clin Proc 2010; 85: 618-29.

5. Teicher MH, Samson JA, Childhood Maltreatment and Psychopathology: A case for ecophenotypic variants as clinically and neurobiologically distinct subtypes Am J Psychiatry 2013; 170: 1114-33.

6. Kendler KS, Bulik CM, Silberg J, Hettema JM, Myers J, Prescott CA. Childhood sexual abuse and adult psychiatry and substance use disorders in women. An epidemiologic and cotwin control analysis. Arch Gen Psychiatry 2000; 57: 953-9.

7. Krug EG, Dahlberg LL, Mercy JA, Zwi AB. World report on violence and health. Geneva: World Health Organization, 2002.

8. Goldman JDG, Padayachi UK. Some methodological problems in estimating incidence and prevalence in child sexual abuse research. J Sex Research 2000; 37: 305-14.

9. Fallon B, Trocme N, Fluke J, MacLaurin B, Tommy L, Yuan YY. Methodological challenges in measuring child maltreatment. Child Ab Neglect 2009; 34: 70-9.

10. Geraerts E, Schooler JW, Merckelbach H, Jelicic M, Hauer BJ, Ambadar Z. The reality of recovered memories: corroborating continuous and discontinuous memories of childhood sexual abuse. Psychol Sci 2007; 18: 564-8.

11. Boakes J. False complaints of sexual assault: recovered memories of childhood sexual abuse. Med Sci Law 1999; 39: 112-20.

12. Dallam SJ. Crisis or creation? A systematic examination of "false memory syndrome". J Child Sex Abus 2000; 9: 9-36.

13. Eissler KR. Freud and the seduction theory: A brief love affair. Madison: International Universities Press, 2001.

14. Masson JM. The assault on truth (Freud's suppression of the seduction theory). New York: Ferrar, Straus and Giroux, 1984.

15. Israëls H. Der Fall Freud: die Geburt der Psychoanalyse aus der Lüge. Hamburg: Europäische Verlagsanstalt, 1999.

16. Ricouer P. De l'interpretation: Essai sur Freud. Paris: Editions du Seuil, 1965.

17. Freud S. Die Abwehr-Neuropsychosen. Versuch einer psychologischen Theorie der adquierierten Hysterie, vieler Phobien und Zwangsvorstellungen und gewisser halluzinatorischer Psychosen. Gesammelte Werke I; 1894: 57-73.

18. Freud S. Über die Berechtigung, von der Neurasthenie einen bestimmten Symptomenkomplex als "Angstneurose" abzutrennen. Gesammelte Werke I; 1895: 311-41.
19. Freud S. Weitere Bemerkungen über die Abwehr-Neuropsychosen. Gesammelte Werke I; 1896: 377-403.

20. Freud S. L’hérédité et l'étiologie des névroses. Gesammelte Werke I; 1896: 404-22.

21. Freud S. Zur Ätiologie der Hysterie. Gesammelte Werke I; 1896: 423-59.

22. Freud S, Breuer J. Studien über Hysterie. Frankfurt: Fischer, 1970.

23. Freud S. Briefe an Wilhelm Fließ 1887-1904. Frankfurt: Fischer, 1985.

24. Anzieu D. L'autoanalyse de Freud et la découverte de la psychoanalyse. 3em éd. Paris: Presses Universitaires de France, 1998.

25. Grinstein A. On Sigmund Freud's dreams. $2^{\text {nd }}$ ed. Madison, CT: International Universities Press, 1980.

26. Ahbel-Rappe K. "I no longer believe": did Freud abandon the seduction theory? J Amer Psychoanal Ass 2006; 54: 171-99.

27. Esterson A. The mythologizing of psychoanalytic history: deception and self-deception in Freud's accounts of the seduction theory episode. Hist Psychiat 2001; 4: 329-52.

28. Freud S. Die Sexualität in der Ätiologie der Neurosen. Gesammelte Werke I; 1898: 489-515.

29. Freud S. Die Traumdeutung. Gesammelte Werke II/III; 1900: 1-642.

30. Freud S. Zur Psychopathologie des Alltagsleben. Gesammelte Werke IV: 1901: 1-310.

31. Freud S. Drei Abhandlungen zur Sexualtheorie. Gesammelte Werke V; 1905: 27-145.

32. Freud S. Meine Ansichten über die Rolle der Sexualität in der Ätiologie der Neurosen. Gesammelte Werke V; 1906: 147-59.

33. Freud S. Zur Geschichte der psychoanalytischen Bewegung. Gesammelte Werke X; 1914: 43-113.

34. Freud S. Selbstdarstellung. Gesammelte Werke XI; 1925: 31-96.

35. Freud S. Aus der Geschichte einer infantilen Neurose. Gesammelte Werke XII; 1918: 27-157.

36. Freud S. Vorlesungen zur Einführung in die Psychoanalyse. Gesammelte Werke XI; 1916-1917: 1-482.

37. Freud S. Der Mann Moses und die monotheistische Religion. Gesammelte Werke XVI; 1939: 101-246.

38. Freud S. Abri $\beta$ der Psychoanalyse. Gesammelte Werke XVII; 1940: 63-138

39. American Psychiatric Association. Diagnostic and Statistical Manual of Mental Disorders. $4^{\text {th }}$ edition. Text Revision (DSM-IV-TR). Washington: American Psychiatric Press, 2000.

40. Freud S. Konstruktionen in der Analyse. Gesammelte Werke XVI; 1937: 41-56. 